\title{
Avaliação das Inteligências Múltiplas em Crianças do Ensino Fundamental
}

\author{
Margarida Pocinho ${ }^{1, *}$ (1) \& Cristina $\operatorname{Mendes}^{2}$ (1) \\ ${ }^{1}$ Universidade da Madeira \& Centro de Investigação em Turismo, Sustentabilidade e Bem-Estar, \\ Universidade do Algarve, Faro, Portugal \\ ${ }^{2}$ Universidade da Madeira, Funchal, Portugal
}

\begin{abstract}
RESUMO - Este estudo pretende avaliar as inteligências múltiplas das crianças portuguesas em idade escolar através do Multiple Intelligence Survey for Kids de Laura Candler. Após tradução e retrotradução e da respetiva validação junto de experts, o instrumento foi aplicado a 112 crianças do ensino fundamental público ( $1^{\circ}$ ciclo do ensino básico), dos 6 aos 10 anos, $61 \%$ do sexo masculino. Os resultados indicam que as inteligências com valores mais elevados são a visuo-espacial, corporal-cinestésica, naturalística e interpessoal. Não existem diferenças de género exceto nas inteligências linguísticoverbal e musical, com as meninas a apresentarem valores superiores aos meninos. Os dados mostram a existência de todas as inteligências propostas por Howard Gardner e os perfis variam de criança para criança.
\end{abstract}

PALAVRAS-CHAVE: Inteligências Múltiplas, Ensino Fundamental, avaliação

\section{Primary School Children's Multiples Intelligences Assessment}

\begin{abstract}
This study aims to evaluate Portuguese primary school children multiple intelligences. The participants were 112 children from public Portuguese schools, aged from six to ten years old, $61 \%$ male. The instrument used was the Portuguese adaptation of Laura Candler Multiple Intelligence Survey for Kids. After translation and retro translation, the scale was applied to the children, individually or in small groups. The results show that visuospatial, kinesthetic, naturalistic and interpersonal are the intelligences with higher values. There are no differences between gender except on linguisticverbal and musical intelligences, with the girls presenting higher values. Data show the existence of all intelligences proposed by Howard Gardner, with variations among the children.
\end{abstract}

KEYWORDS: Multiple Intelligences, Primary School, assessment

A inteligência foi encarada e medida durante muitos anos unicamente através das capacidades cognitivas apresentadas pelo indivíduo, pelo que estava assim, consignada somente aos seus aspetos teóricos e às capacidades ensinadas $\mathrm{e}$ utilizadas nas escolas. Há relativamente poucos anos, alguns autores começaram a colocar em causa esta teoria, defendendo que a inteligência englobava muito mais do que apenas estas capacidades, particularmente quando pensamos que pessoas consideradas bem-sucedidas não possuem obrigatoriamente níveis elevados de inteligência (Pocinho, 2010).

Tendo em conta as teorias que defendem que a inteligência opera da mesma maneira em todas as pessoas, torna-se relativamente complexo explicar o fato de crianças com níveis de inteligência semelhantes apresentarem, muitas vezes, dificuldades na aquisição dos mesmos conhecimentos. Ainda mais difícil é explicar o porquê de crianças com elevados níveis de inteligências apresentarem dificuldades de aprendizagem e, o fato de a superdotação se apresentar, por vezes, apenas numa determinada área da inteligência (Pocinho, 2009).

Apesar de algum consenso científico de que a inteligência envolve muito mais do que aquilo que se acreditava estar num único fator, o designado Quociente Intelectual (QI), infelizmente não têm sido tomadas medidas para que a escola atual se aproxime desta constatação e responda às necessidades específicas de cada aluno (Branco, 2003). $\mathrm{O}$ estudo da inteligência tem suscitado vasto debate entre

*E-mail: mpocinho@staff.uma.pt

- Submetido: 16/11/2017; Aceito: 25/12/2020. 
os especialistas, os quais dividem as suas opiniões sobre a forma como esta funciona. Enquanto alguns acreditam que a inteligência possui um único fator, outros acreditam que esta é composta por uma multiplicidade de fatores. Defendendo este ponto encontra-se o psicólogo americano Howard Gardner, que desenvolveu a teoria das Inteligências Múltiplas, objeto do nosso estudo.

\section{Em Torno do Conceito de Inteligência}

O estudo da inteligência remonta a Wilhelm Wundt e aos seus estudos no Laboratório de Psicologia Experimental de Leipzig (Almeida et al., 2012; Candeias \& Almeida, 2007). Contudo, os primeiros estudos sistematizados deste constructo são atribuídos a Sir Francis Galton (Almeida, 2002; Almeida et al., 2012), que definiu a inteligência em termos de energia e sensibilidade aos estímulos físicos. Foi um dos primeiros autores a ter em conta as diferenças individuais entre sujeitos, bem como a valorizar o fator hereditário das mesmas (Schultz \& Schultz, 2007).

Mas só em 1905 é que Alfred Binet e Théodore Simon criaram o primeiro instrumento verdadeiramente psicológico que permitia medir a inteligência, designado por Escala de Inteligência de Binet-Simon (Almeida, 2002; Almeida et al., 2012; Schultz \& Schultz, 2007). Todavia, uma vez que este instrumento se concentrava nas funções cognitivas, acabou por associar a inteligência às capacidades escolares, marcando fortemente, e ao longo de vários séculos, a forma como os investigadores consideraram a inteligência. Ainda assim, a criação deste teste, permitiu a introdução do conceito de idade mental que deu origem à tradição psicométrica e permitiu a medida e quantificação da inteligência, atribuindo ao sujeito um papel ativo.

A Escala de Inteligência de Binet-Simon foi revista por diversos autores, sendo traduzida por Henry Goddard, em 1908, do francês para o inglês (Schultz \& Schultz, 2007) e adaptada, em 1916, por Lewis Terman, passando a ser conhecida por Escala Stanford-Binet (Almeida, 2002; Schultz \& Schultz, 2007). Este autor foi a primeira pessoa a utilizar o Quociente de Inteligência para cotar os resultados. Em 1939, David Wechsler desenvolveu o primeiro teste de inteligência a incluir testes não-verbais, que acabou por se tornar o mais popular e mais utilizado teste de inteligência (Almeida, 2002). Contrariamente ao que acontecia na Europa, nos Estados Unidos, alguns psicólogos, incluindo Louis Leon Thurstone e Joy Paul Guilford, acreditavam que a inteligência englobava um conjunto de diversos fatores, relativamente independentes entre si (Almeida, 2002).

\section{Da Inteligência às Inteligências Múltiplas}

A teoria das Inteligências Múltiplas, como o próprio Howard Gardner admite, abrange essencialmente uma sistematização das teorias de Thurstone e Guilford, e foi divulgada pela primeira vez em 1983 no seu livro Frames of Mind. Segundo Gardner (1983), a inteligência é descrita como "a capacidade para resolver problemas ou criar produtos que sejam úteis num ou mais contextos culturais" (p. 11).

O grande mérito desta teoria é a passagem do paradigma unidimensional para multidimensional (Gaspári \& Schwarts, 2002), que considera a inteligência como um conjunto de diferentes habilidades, distintas e relativamente independentes entre si (Amiryousefi \& Zarei, 2011; Pocinho, 2009), mas que trabalham em conjunto (Diaz \& HeiningBoynton, 1995).

Neste contexto Gardner $(1983,2006)$ definiu oito inteligências. Assim, a inteligência musical envolve a sensibilidade, o reconhecimento e a habilidade para interpretar, compor, reconhecer ou simplesmente apreciar padrões e elementos rítmicos e melódicos; a inteligência corporal-cinestésica corresponde à utilização do corpo e do movimento para expressar pensamentos e sentimentos, representar objetos, ações ou até conceitos abstratos, e utilizar imagens mentais para coordenar movimentos corporais; a inteligência visuo-espacial equivale à consciência do mundo visual e engloba a sensibilidade à cor, ao desenho e à forma, e à capacidade para manipular e criar imagens mentais, ao relacionar padrões e observar semelhanças nas formas espaciais; a inteligência lógico-matemática diz respeito ao raciocínio lógico-dedutivo, ou seja, à correta utilização dos números e dos elementos matemáticos, à habilidade para deduzir conclusões lógicas e identificar relações de causa e efeito; a inteligência linguístico-verbal abrange a escrita, a leitura e a fala, através da mestria da linguagem e da correta utilização desta e das palavras, nos seus diferentes domínios, semântica, sintaxe e som; a inteligência interpessoal referese à capacidade para compreender e distinguir nos outros as suas intenções, interesses, necessidades, motivações e desejos, facilitando os relacionamentos interpessoais; a inteligência intrapessoal equivale ao autoconhecimento, à capacidade de compreender os sentimentos, emoções, medos, motivações, interesses, necessidades, ideais, pontos fortes e fracos em si próprio, permitindo-lhe dirigir eficazmente os seus esforços e comportamentos; a inteligência naturalística inclui o conhecimento e preocupação pela natureza, a sensibilidade aos acontecimentos que ocorrem na mesma e a capacidade de reconhecer, distinguir e classificar seres vivos e não-vivos; e, por fim, a inteligência existencial procura o sentido da vida e reflete sobre o significado da vida e o sentido da morte. Contudo, Gardner (2006) mantém algumas reservas em confirmar a existência desta última inteligência, devido à falta de evidências claras das estruturas e processos cerebrais dedicados à mesma, pelo que esta nem sempre é incluída como uma das Inteligências Múltiplas.

Estudos neurológicos recentes (e.g. estudo longitudinal de cinco anos levado a cabo pela equipe do Brain and Creativity Institute at the University of Southern California coordenada por António Damásio, com crianças de seis e 
sete anos de idade) revelam a presença de determinadas áreas do cérebro mais propensas para certas formas de cognição (Habibi et. al., 2014). Esta constatação sugere que existem diferentes estruturas neurológicas responsáveis por distintas modalidades de processamento de informação e, por consequência, de mais do que uma única inteligência.

Também Howard Gardner, entre as várias investigações que realizou, estudou o desenvolvimento de diferentes capacidades em crianças normais e superdotadas e a produção intelectual em adultos com lesões cerebrais, de modo a averiguar a relação entre capacidades e zonas do cérebro afetadas (Gardner, 2011a). Este estudo sugeriu a existência de inteligências múltiplas. Para o autor, a inteligência não pode ser centrada numa só dimensão linguística ou lógico-matemática, dadas as diferenças individuais no desempenho de tarefas verbais-académicas, rítmico-motoras ou sociais, entre outras. Gardner (2011b) combateu a ideia unitária de inteligência, definida em termos de testes padronizados de QI, e tentou provar que o indivíduo desenvolve, pelo menos, oito inteligências, como anteriormente foram descritas.

Todas as inteligências são inatas e igualmente importantes, e todos os alunos possuem potencialidades em cada uma delas, independentemente da educação ou da cultura a que pertencem. No entanto, o desenvolvimento de cada inteligência é influenciado quer por fatores hereditários e neurobiológicos, quer por fatores ambientais. Gardner (2006, 2011a, 2011b) coloca a hipótese do desenvolvimento de alguns talentos só ser possível quando integrados num ambiente sociocultural que os promova. Embora valorize o contexto sociocultural das múltiplas inteligências, uma vez que cada cultura tende a enfatizar inteligências particulares, Gardner é insensível às diferenças entre contextos socioculturais.

Desta forma, Gardner explica também o porquê de algumas pessoas parecerem mais inteligentes do que outras. É que a estimulação tem uma grande influência no desenvolvimento de cada uma das inteligências e a promoção de uma delas melhora a inteligência global da pessoa, devido à interdependência entre as inteligências múltiplas (Gaspári \& Schwarts, 2002).

Qualquer uma das inteligências tem a sua forma própria de processamento de informação, além de envolver um sistema simbólico próprio que estabelece o contacto entre os aspetos básicos da cognição e a variedade de papéis e funções culturais. No entanto, embora estas inteligências sejam, até certo ponto, independentes umas das outras (o que justifica diferentes níveis entre as inteligências), elas raramente funcionam de forma isolada. Em termos práticos, a grande maioria das tarefas a serem desempenhadas pelo aluno ilustram bem a necessidade de uma combinação de inteligências (Gardner, 2011a, 2011b).

\section{Ensino-Aprendizagem Tendo em conta as Inteligências Múltiplas}

Pesquisas cognitivas recentes documentam que os alunos possuem diferentes tipos de capacidades intelectuais; por consequência, aprendem, memorizam e apresentam desempenhos diferenciados e compreendem de modos diferentes. Nesta perspectiva, o professor deve ser alguém que adquiriu uma compreensão profunda (deep understanding) dos conhecimentos que pretende ensinar, com a capacidade de abordar os temas de múltiplas perspetivas. Para além disso, este professor deve educar para a compreensão profunda e isso só se consegue, com pedagogias diferenciadas que recorram a várias maneiras de abordar o mesmo tema. É esta abordagem multidisciplinar que Gardner (2011b) recomenda às escolas como uma forma de facilitar, não só uma melhor compreensão, mas também uma melhor transferência de saberes.

A investigação tem mostrado o sucesso da abordagem das múltiplas inteligências na aprendizagem escolar, pois cada aluno possui um perfil único de inteligências.

Um estudo longitudinal de 2011 a 2015, levado a cabo por Lai e Yap (2016), mostrou que a aplicação da teoria das múltiplas inteligências capacita estratégias mais estruturadas que melhoram significativamente as competências cognitivas dos alunos na aprendizagem das ciências. O perfil de inteligências múltiplas mostrou uma passagem da dominância da inteligência intrapessoal nos primeiros anos do estudo (2011-2013) para as inteligências interpessoal, cinestésica e naturalística nos dois últimos anos (2014-2015).

Outra investigação mostra um aumento do rendimento acadêmico e da retenção do conhecimento (Yalmanci \& Gozum, 2013). Esses autores realizaram uma investigação experimental com uma amostra aleatória de 60 estudantes do $3^{\circ}$ ano do Departamento de Ciências da Faculdade de Educação duma universidade turca. Trinta estudantes foram sujeitos ao método das "inteligências múltiplas" (grupo experimental), durante um ano letivo, e os outros 30 estudantes (grupo de controle), sujeitos ao método tradicional. O grupo experimental obteve ganhos significativos nas classificações e na aquisição/retenção de conhecimento, quando comparado com o grupo de controle.

\section{Avaliação das Inteligências Múltiplas}

A teoria das inteligências múltiplas, devido à grande ênfase atribuída aos aspetos contextuais e criativos de cada inteligência, cria dificuldades na criação de um instrumento que permita a sua medição. Alguns autores criaram instrumentos que têm cumprido este propósito. 
Howard Gardner aconselha a utilização do Multiple Intelligences Developmental Assessment Scales (MIDAS), um questionário de autorrelato que avalia a predisposição intelectual do indivíduo para cada uma das oito inteligências múltiplas (excluindo a inteligência existencial que na altura ainda não havia sido comprovada). É composto por escalas principais, representativas de cada inteligência e por subescalas que descrevem atividades e capacidades específicas, servindo como indicadores qualitativos da capacidade e facilitadores da interpretação dos resultados (Shearer, 2009, 2012).

A autora Sue Teele (1995) criou um instrumento denominado de Teele Inventory of Multiple Intelligences (TIMI), desenvolvido em 1992, que pode ser utilizado a partir dos três anos de idade. Este inventário contém 28 perguntas, com duas opções de resposta representadas por pandas que caracterizam cada uma das sete inteligências múltiplas primeiramente descritas por Gardner (Oteng, 2012; Sahli et al., 2011).

No Brasil, foi também criado um instrumento designado de Questionário Icônico, composto por 24 itens, representativos das inteligências múltiplas, em que o indivíduo deve pontuar, de acordo com o grau de autoidentificação com cada uma delas. A par deste questionário costuma também ser aplicado o Questicônico, consistindo na seleção de cinco dos 24 itens representativos das inteligências múltiplas e na elaboração de uma história com eles. A análise é feita através da apreciação dos ícones escolhidos e da identificação das inteligências predominantes no contexto da história (Zandomeneghi, 2005).

Laura Candler criou o Multiple Intelligence Survey for Kids, especialmente destinado às crianças. Este se divide em 24 afirmações que a criança deve classificar, de 0 a 5 , de acordo com o seu grau de concordância. $\mathrm{O}$ resultado máximo que pode ser conseguido numa inteligência é de 15. Este instrumento foi criado com o intuito de identificar os pontos fortes e as potencialidades das crianças. A sua elaboração deveu-se ao fato de a maior parte das ferramentas existentes para medir as inteligências múltiplas serem demasiado longas e complexas para crianças. Contudo, a autora alerta para a ausência de qualquer investigação científica e consequentemente para a utilização cuidadosa do mesmo (Candler, 2011). Uma vez que este instrumento está mais focado nas crianças e é mais simples, em termos de compreensão e preenchimento, foi este o escolhido para ser utilizado nesta investigação.

Tendo em conta o exposto, após explanar alguns conceitos-base em torno da(s) inteligência(s) e da sua avaliação, o principal objetivo deste estudo é avaliar as inteligências múltiplas numa amostra de crianças portuguesas a frequentar o primeiro ciclo do ensino básico, com idades entre os seis e os dez anos. Não havendo nenhum instrumento para avaliá-las, decidiu-se adaptar o Multiple Intelligence Survey for Kids, de Laura Candler, com a respectiva autorização da autora, para a população portuguesa e depois realizar a avaliação das inteligências das crianças.

\section{MÉTODO}

\section{Participantes}

A amostra foi selecionada aleatoriamente, após consentimento informado dos encarregados de educação das crianças. Participaram 112 crianças, 68 (61\%) do sexo masculino, com idades entre os seis e os dez anos, distribuídas pelos quatro anos do ensino fundamental de escolas públicas portuguesas (Tabela 1).

\section{Instrumento}

Os dados foram recolhidos através da adaptação do Multiple Intelligence Survey for Kids, de Laura Candler, traduzido e retrotraduzido para a Língua Portuguesa por duas professoras licenciadas em Inglês (Escala de Avaliação das Inteligências Múltiplas - EAIM). Posteriormente, o instrumento foi validado por três professoras universitárias
Tabela 1

Características dos Participantes

\begin{tabular}{lccc}
\hline \multirow{2}{*}{ Gênero } & & Frequência & \% \\
\hline \multirow{4}{*}{ Idade } & Meninos & 68 & 60,7 \\
& Meninas & 44 & 39,3 \\
\hline & 7 & 13 & 11,6 \\
& 8 & 22 & 19,6 \\
Série & 9 & 29 & 32,1 \\
& 10 & 11 & 25,9 \\
& $1^{\text {a }}$ & 17 & 9,8 \\
\hline & $2^{\text {a }}$ & 38 & 15,2 \\
& $3^{\text {a }}$ & 25 & 33,9 \\
& $4^{\text {a }}$ & 32 & 22,3 \\
\hline
\end{tabular}


da área da Psicologia. A escala é composta por 24 itens referentes às oito inteligências de Gardner. A resposta aos itens é de tipo Likert, com seis opções de resposta $(0=N a ̃ o$ Tem Nada a Ver Comigo; 1=Tem Muito Pouco a Ver Comigo; 2=Tem Pouco a Ver Comigo; 3=Tem Mais ou Menos a Ver Comigo; 4=Tem Muito a Ver Comigo; 5=Tem Tudo a Ver Comigo).

Após análise fatorial com rotação Varimax, com o propósito de averiguar se existiam itens que se agrupassem em fatores, o mesmo não se verificou. Como escala global, as características psicométricas da escala são boas, com um alfa de Cronbach de 0,72, o que lhe confere consistência interna e fiabilidade. Este questionário pode ser aplicado individualmente ou em grupo, demorando o seu preenchimento, entre 20 a 30 minutos (Figura 1).

\section{Procedimento}

Após autorização oficial das Escolas e do consentimento informado dos encarregados de educação das crianças, aplicou-se a escala de avaliação das inteligências múltiplas às crianças que tinham autorização para participar neste estudo. A aplicação foi feita individualmente às crianças que ainda não sabiam ler e em pequenos grupos de cinco alunos às crianças que já sabiam ler. Após a coleta dos dados, estes foram inseridos no programa estatístico SPSS, Versão 22.0, e foi efetuada a sua análise estatística.

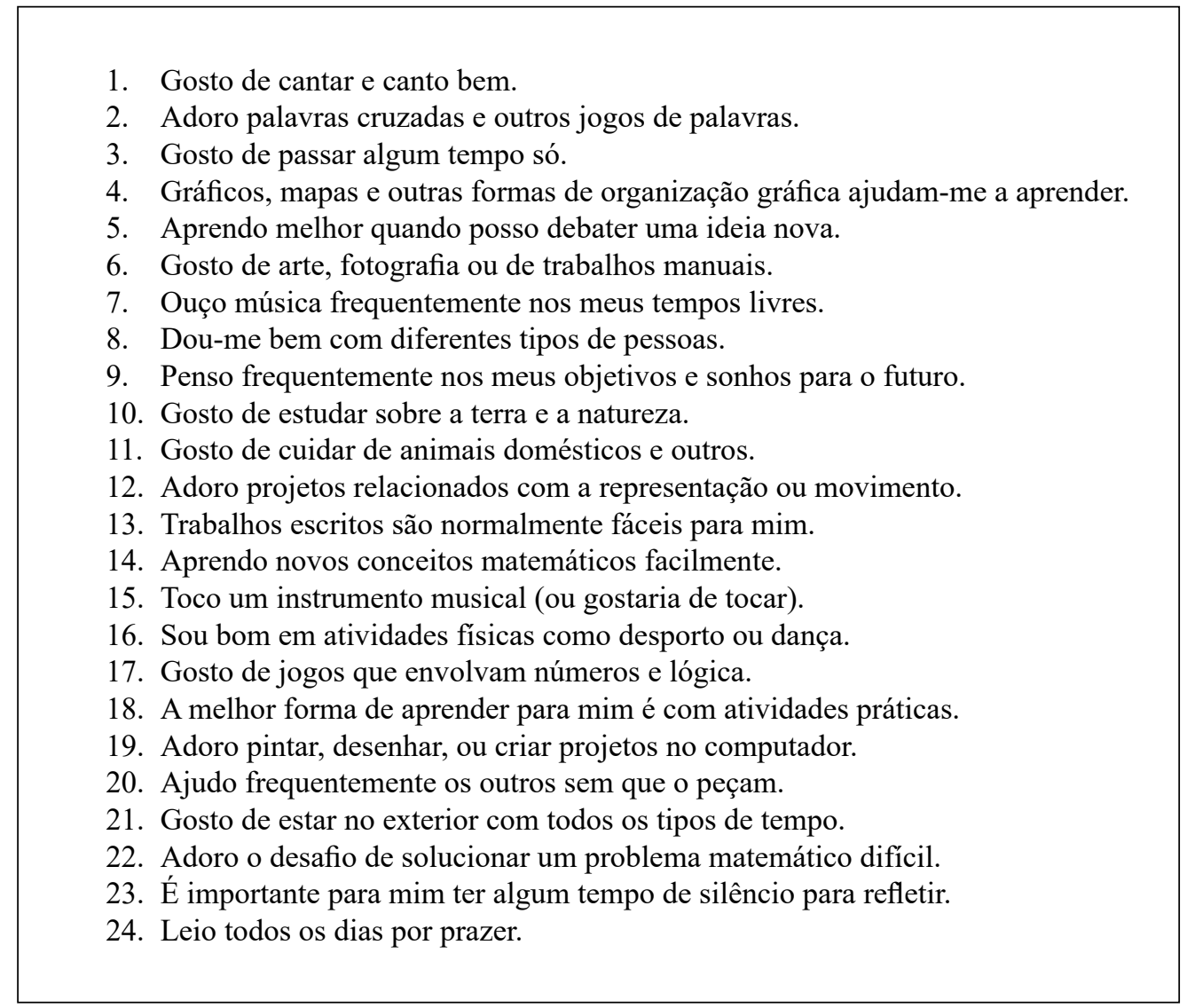

Figura 1. Escala de Avaliação das Inteligências Múltiplas - EAIM (versão experimental)

\section{RESULTADOS}

A tabela 2 apresenta a estatística descritiva de tendência central dos valores obtidos pelos participantes em cada uma das inteligências. Em relação ao valor mínimo, as inteligências visuo-espacial e lógico-matemática são as únicas em que alguns participantes obtiveram zero pontos, correspondente à pontuação mínima que o instrumento permite conceder. A segunda inteligência menos presente nas respostas das crianças é a intrapessoal, seguindo-se das inteligências linguístico-verbal e musical, enquanto a inteligência corporal-cinestésica situa-se na quarta posição. No topo, situam-se a inteligência naturalística e interpessoal. Ao nível do valor máximo, todas as Inteligências Múltiplas alcançaram a pontuação máxima, ou seja, 15 pontos. Em relação às pontuações médias de cada uma das Inteligências 
Múltiplas, por ordem crescente, a inteligência menos pontuada foi a musical $(M=10,6)$, seguida da intrapessoal ( $M$ $=10,7)$, linguístico-verbal $(M=11,47)$, lógico-matemática $(M=11,54)$, interpessoal $(M=12,05)$, naturalística $(M=$ $12,09)$, corporal-cinestésica $(M=12,21)$ e finalmente, a inteligência mais valorizada, a inteligência visuo-espacial $(M$ $=12,22$ ). O desvio-padrão para cada uma das inteligências varia entre os 2,55 e os 3,68.

Não existem diferenças de gênero exceto nas inteligências linguístico-verbal $\left(t_{(110)}=-2,44, p=0,016\right)$ e musical $\left(t_{(110)}\right.$
$=-2,5, p=0,016)$, com as meninas a apresentarem valores superiores aos meninos.

Através da ANOVA e do teste pos-hoc de Bonferroni, apuraram-se as diferenças das pontuações obtidas consoante a série de escolaridade. Existem diferenças consoante à série escolar nas inteligências linguístico-verbal $\left(F_{(3,111)}=6,38\right.$, $\left.p=0,001 ; 2^{\mathrm{a}}>4^{\mathrm{a}}\right)$, lógico-matemática $\left(F_{(3,111)}=7,22, p=\right.$ 0,$\left.004 ; 2^{\mathrm{a}}>4^{\mathrm{a}}\right)$, interpessoal $\left(F_{(3,111)}=4,53, p=0,005 ; 2^{\mathrm{a}}>\right.$ $\left.4^{\mathrm{a}}\right)$, e intrapessoal $\left(F_{(3,111)}=6,18, p=0,001 ; 2^{\mathrm{a}}, 3^{\mathrm{a}}\right.$ e $\left.4^{\mathrm{a}}>1^{\mathrm{a}}\right)$.

A distribuição dos dados obtidos na EAIM por percentis encontra-se na Tabela 3.

Tabela 2

Estatística Descritiva dos Resultados Obtidos na EAIM

\begin{tabular}{lcccc}
\hline Inteligência & Mín. & Máx. & $\boldsymbol{M}$ & DP \\
\hline Linguística-Verbal & 2,00 & 15,00 & 11,47 & 2,93 \\
Visuo-Espacial & 0,00 & 15,00 & 12,22 & 2,66 \\
Lógico-Matemática & 0,00 & 15,00 & 11,54 & 3,64 \\
Musical & 2,00 & 15,00 & 10,67 & 3,35 \\
Corporal-Cinestésica & 3,00 & 15,00 & 12,21 & 2,68 \\
Naturalística & 4,00 & 15,00 & 12,10 & 2,58 \\
Interpessoal & 5,00 & 15,00 & 12,05 & 2,57 \\
Intrapessoal & 1,00 & 15,00 & 10,71 & 3,24 \\
\hline
\end{tabular}

Tabela 3

Distribuição dos Resultados Obtidos na EAIM por Percentis

\begin{tabular}{lccc}
\hline & & Percentis & $\mathbf{7 5}^{\mathbf{0}}$ \\
\cline { 2 - 4 } & $\mathbf{2 5}^{\mathbf{0}}$ & $\mathbf{5 0}^{\mathbf{0}}$ (Mediana) & 14,00 \\
\hline Linguística-Verbal & 10,00 & 12,00 & 14,00 \\
Visuo-Espacial & 10,00 & 13,00 & 15,00 \\
Lógico-Matemática & 9,00 & 12,00 & 13,75 \\
Musical & 9,00 & 11,00 & 14,75 \\
Corporal-Cinestésica & 10,00 & 13,00 & 15,00 \\
Naturalística & 10,00 & 12,50 & 15,00 \\
Interpessoal & 10,00 & 12,00 & 13,00 \\
Intrapessoal & 9,00 & 11,00 & \\
\hline
\end{tabular}

\section{DISCUSSÃO}

O principal objetivo desta investigação é avaliar as inteligências múltiplas das crianças do ensino fundamental. Genericamente, os resultados mostram que as crianças não possuem uma determinada inteligência manifestamente dominante sobre as demais, ou seja, não podemos afirmar a existência de uma inteligência única, mas sim de um perfil de inteligências, no qual cada criança possui características de todas as inteligências, dando mais ou menos destaque a cada uma delas.
Os dados desta amostra revelam que a inteligência menos presente na mesma é a inteligência musical, enquanto a inteligência mais elevada é a visuo-espacial. Estes dados, quando comparados com os dados obtidos por Beceren (2010), revelam semelhanças ao nível da inteligência mais utilizada e da menos utilizada. Em ambas as amostras, a inteligência mais usada é a visuo-espacial, enquanto a menos utilizada é a musical. No caso de crianças superdotadas, o estudo de Chan (2006) concluiu que a inteligência 
lógico-matemática possuía valores mais elevados do que as restantes inteligências.

No entanto, quando comparamos os dados obtidos com os resultados encontrados por Sahli et al. (2011) com crianças surdas, utilizando o Teele Inventory of Multiple Intelligences, observamos algumas diferenças, nomeadamente no que diz respeito à inteligência mais baixa nestas crianças, que neste caso é a inteligência interpessoal, seguida da inteligência linguístico-verbal, enquanto a inteligência mais alta é a visuo-espacial. No entanto, estas diferenças são já esperadas, devido às especificidades destas crianças.

Em relação às diferenças entre gênero, as meninas obtiveram valores mais elevados na grande maioria das inteligências múltiplas, destacando-se as inteligências linguístico-verbal e musical. Neste caso, os resultados desta amostra coincidem com os resultados do estudo de Elibol e Tugrul (2001), citados por Beceren (2010), que também concluíram que as meninas possuem maior inteligência linguístico-verbal e musical do que os rapazes. Contudo, não encontraram diferenças significativas em relação às restantes inteligências. Também Llor et al. (2012), ao compararem as inteligências múltiplas dos alunos superdotados e o gênero dos mesmos, descobriram que as meninas possuem valores mais elevados nas inteligências linguístico-verbal, musical e visuo-espacial, enquanto os rapazes possuem valores mais elevados nas inteligências lógico-matemática e naturalística. Estes resultados coincidem, em parte, com os dados obtidos, especialmente no que diz respeito à inteligência linguísticoverbal e musical.

Contudo, os resultados dos estudos já realizados dividem-se. Investigadores, como Sözen et al. (2009) e Serin et al. (2009), afirmam não haver encontrado quaisquer diferenças significativas entre gêneros. Mas no caso de Chen (2004), os dados encontrados diferem bastante dos encontrados neste estudo, uma vez que este autor encontrou diferenças entre rapazes e meninas, a favor dos rapazes, que tinham maior inteligência lógico-matemática, enquanto as meninas possuíam apenas maior inteligência interpessoal. Loori (2005) concluiu que homens e mulheres preferem atividades diferentes para facilitar a sua aprendizagem.

A relação entre o gênero feminino e as inteligências múltiplas foi estudada por Al-Sulim (2012), que ordenou as Inteligências Múltiplas utilizadas pelas mulheres: lógico-matemática, interpessoal, visuo-espacial, corporalcinestésica, linguístico-verbal, intrapessoal, naturalística e existencial. Estes resultados diferem muito dos resultados encontrados neste estudo. No entanto, é de realçar que a idade dos participantes em cada uma das amostras é muito diferente, pelo que a comparação feita entre os mesmos tem de ser considerada com muito cuidado.

Ao refletirmos sobre os anos de escolaridade, encontramos diferenças mais significativas entre a $1^{\mathrm{a}}$ série e as restantes turmas desta amostra. Assim, os estudos de Yussen e Kane (1985) e Stipek e Gralinski (1996) vão também neste sentido, uma vez que os mesmos descobriram que a idade das crianças é um fator significativo na forma como a sua inteligência funciona. Também Almeida et al. (2009) descobriram diferenças entre crianças mais novas e mais velhas, ao nível das inteligências linguístico-verbal, corporal-cinestésica, visuo-espacial e lógico-matemática. Estes resultados se assemelham aos obtidos no presente estudo, uma vez que as diferenças mais significativas, ao nível dos anos de escolaridade, dizem respeito também às inteligências linguístico-verbal e lógico-matemática. Elibol (2000) descobriu ainda que as crianças mais novas usam mais a inteligência visuo-espacial, seguida da inteligência corporal-cinestésica e da inteligência intrapessoal, comprovando novamente os resultados obtidos. Também Teele (1995) chegou às mesmas conclusões, mas no que diz respeito à terceira inteligência mais utilizada. A autora descobriu que os meninos preferem utilizar a inteligência lógico-matemática, enquanto as meninas preferem a inteligência linguístico-verbal.

No nosso estudo foi ainda possível observar que as inteligências múltiplas estão correlacionadas entre si, de forma mais ou menos significativa. Assim, podemos confirmar a ideia defendida por Gardner de que as Inteligências Múltiplas estão correlacionadas umas com as outras. Estes dados são também confirmados pelos estudos de Beceren (2010) e Behjat (2012), os quais mesmo trabalhando com amostras de idades bastante diferentes chegaram à mesma conclusão. Savas (2012) confirmou também esta conclusão e refere que os seus resultados são semelhantes aos de outros estudos, como Moran et al. (2006) e Torresan (2007).

Os resultados obtidos nesta investigação nos permitem tirar algumas implicações.

A primeira deriva da confirmação, tal como apresentado na teoria de Gardner (1983, 2006), que as inteligências múltiplas estão presentes em todas as crianças, de diferentes formas. Os resultados indicam que as inteligências com valores mais elevados são a inteligência visuo-espacial, corporal-cinestésica, naturalística e interpessoal. Estes dados confirmam a existência de todas as inteligências propostas por Gardner, com variações de pessoa para pessoa, reforçando a importância e necessidade de valorizar as diferentes capacidades apresentadas pelos indivíduos. Se continuarmos com o modelo tradicional de educação utilizado pela escola atual, que valoriza as inteligências linguístico-verbal e lógico-matemática em detrimento das restantes, o mesmo poderá criar dificuldades a alguns alunos na aquisição de conhecimentos.

A segunda implicação dos resultados é que só existem diferenças entre gêneros nas inteligências linguístico-verbal e musical, com o sexo feminino a apresentar valores mais elevados. Não podemos afirmar que rapazes e moças pensam ou utilizam as suas inteligências múltiplas de forma diferente consoante o seu gênero. 
A terceira implicação deriva da quase inexistência de diferenças significativas da distribuição das inteligências múltiplas consoante os diferentes anos de escolaridade das crianças.

A quarta e última implicação é relativa à validade do instrumento. Trata-se de uma escala confiável, que possibilita a avaliação das inteligências múltiplas e permite conhecer as capacidades das crianças. A utilização de tal instrumento pelos professores e psicólogos educacionais pode constituir uma mais-valia para o processo de ensino/ aprendizagem. Possibilitaria adaptar as estratégias e conteúdos da aprendizagem aos pontos fortes das crianças, mas também o reconhecimento dos pontos mais fracos, permitindo o treino e desenvolvimento destes últimos. A escala apresenta boas caraterísticas psicométricas, o que significa que este é instrumento fiável para ser utilizado na nossa população. Contudo, e tendo em conta que a escala foi aplicada apenas a 112 participantes, é necessário aumentar a amostra e realizar estudos em todas as regiões do país, incluindo os arquipélagos da Madeira e Açores, para confirmar os resultados encontrados nesta investigação e para que a aplicação do instrumento possa ser generalizada à população portuguesa.

\section{REFERÊNCIAS}

Almeida, L. (2002). As aptidões na definição e avaliação da inteligência: $\mathrm{O}$ concurso da análise fatorial. Paidéia, 12(23), 5-17. https://doi.org/10.1590/S0103-863X2002000200002

Almeida, L., Ferrando, M., Ferreira, A. I., Prieto, M. D., Fernández, M. C., \& Sainz, M. (2009). Inteligências múltiplas de Gardner: É possível pensar a inteligência sem um fator g? Psychologica, 50, 41-55. https://doi.org/doi:10.14195/1647-8606_50_3.

Almeida, L., Roazzi, A., \& Spinillo, A. (2012). O estudo da inteligência: Divergências, convergências e limitações dos modelos. Psicologia: Teoria e Pesquisa, 5(2), 217-230. https:// periodicos.unb.br/index.php/revistaptp/article/view/17070

Al-Sulim, H. S. (2012). Prediction of the correlation between the strategies of the teaching methods and the multiple intelligence of some graduate females tudents at Imam Mohammad Ibn Saud Islamic University. Procedia - Social and Behavioral Sciences, 47, 1268-1275. https://doi.org/10.1016/j.sbspro.2012.06.810

Amiryousefi, M., \& Zarei, G. R. (2011). Are MI and motivation catered for in EAP textbooks? Procedia - Social and Behavioral Sciences, 30, 573-577. https://doi.org/10.1016/j. sbspro.2011.10.111

Beceren, B. C. (2010). Determining multiple intelligences preschool children (4-6 age) in learning process. Procedia Social and Behavioral Sciences, 2, 2473-2480. https://doi. org/10.1016/J.SBSPRO.2010.03.356

Behjat, F. (2012). Interpersonal and intrapersonal intelligences: Do they really work in foreign-language learning? Procedia - Social and Behavioral Sciences, 32, 351-355. https://doi. org/10.1016/j.sbspro.2012.01.052

Branco, A. (2003). Para além do QI. Quarteto Editora.

Candeias, A. A., \& Almeida, L. S. (Coords.). (2007). Inteligência humana: Investigação e aplicações (Vol. 1). Quarteto Editora.

Candler, L. (2011). Multiple Intelligence survey for kids. http:// www.lauracandler.com/free/misurvey

Chan, D. W. (2006). Perceived Multiple Intelligences among male and female Chinese gifted students in Hong Kong: The structure of the student Multiple Intelligences Profile. Gifted Child Quarterly, 50(4), 325-338. https://doi. org/10.1177/001698620605000405

Chen, J. Q. (2004). Theory of multiple intelligences: Is it a scientific theory? Teachers College Record, 106, 17-23. http://dx.doi. org/10.1111/j.1467-9620.2004.00313.x

Diaz, L., \& Heining-Boynton, A. L. (1995). Multiple intelligences, multiculturalism, and the teaching of culture. International Journal of Educational Research, 23(7), 607-617. https://doi. org/10.1016/0883-0355(96)80440-X

Elibol, F. (2000). The tendency of intelligence usage in the scope of Multiple Intelligence theory with the six-year-old preschool children [Tese não publicada]. Hacettepe University].
Gaspári, J. C., \& Schwarts, G. M. (2002). Inteligências múltiplas e representações. Psicologia: Teoria e Pesquisa, 18(3), 261-266. https://doi.org/10.1590/S0102-37722002000300004

Gardner, H. (1983). Frames of mind: The theory of Multiple Intelligences. Basic Books.

Gardner, H. (2006). Multiple intelligences: New horizons. Basic Books.

Gardner, H. (2011a). Frames of mind: The theory of multiple intelligences. Hachette UK.

Gardner, H. (2011b). Leading minds: An anatomy of leadership. Hachette UK.

Habibi, A., Ilari, B., Crimi, K., Metke, M., Kaplan, J. T., Joshi, A. A., ... \& Ficek, B. (2014). An equal start: Absence of group differences in cognitive, social, and neural measures prior to music or sports training in children. Frontiers in Human Neuroscience, 8, 690. https://doi.org/10.3389/ fnhum.2014.00690

Lai, H. Y. \& Yap, S. L. (2016) Application of Multiple Intelligence Theory in the assessment for learning. In: S. Tang \& L. Logonnathan. Assessment for learning within and beyond the classroom. (Eds. pp. 427-436) Springer. https://doi. org/10.1007/978-981-10-0908-2_36

Llor, L., Ferrando, M., Ferrándiz, C., Hernández, D., Sáins, M., Prieto, M. D., \& Fernández, M. C. (2012). Inteligencias Múltiples y alta habilidad. Aula Abierta, 40, (1), 27-38. https:// dialnet.unirioja.es/servlet/articulo?codigo $=3791837$.

Loori, A. A. (2005). Multiple intelligences: A comparative study between the preferences of males and females. Social Behavior and Personality: An international Journal, 33(1), 77-88. https://doi.org/10.2224/sbp.2005.33.1.77

Moran, S., Kornhaber, M., \& Gardner, H. (2006). Orchestrating multiple intelligences. Educational Leadership, 64(1), 22-27. https://www.ascd.org/el/articles/orchestrating-multipleintelligences.

Oteng, E. N. (2012). A comparative analysis of multiple intelligence theory with relationship to gender and grade level in selected schools in Ghana. [Dissertação de mestrado. Mid-America Baptist Theological Seminary]. ProQuest LLC. https://eric.ed .gov/?q=multiple+intelligences\&ff $1=$ subElementary + School +Students\&ff $2=$ pubDissertations $\% 2$ fTheses +-+ Doctoral+Di ssertations\&id=ED550660

Pocinho, M. (2009). Superdotação: Conceitos e modelos de diagnóstico e intervenção psicoeducativa. Revista Brasileira de Educação Especial, 15(1), 3-14. https://doi.org/10.1590/ S1413-65382009000100002

Pocinho, M. (2010). Psicologia, cognição e sucesso escolar: Concepção e validação dum programa de estratégias de 
aprendizagem. Psicologia Reflexão e Crítica, 23(2), 362-373. https://doi.org/10.1590/S0102-79722010000200019

Sahli, S., Laszig, R., Aschendorff, A., Kroeger, S., Wesarg, T., $\&$ Belgin, E. (2011). Comparison of learning preferences of Turkish children who had been applied cochlear implantation in Turkey and Germany according to theory of Multiple Intelligence. International Journal of Pediatric Otorhinolaryngology, 75(12), 1576-1584. https://doi. org/10.1016/j.ijporl.2011.09.011

Savas, P. (2012). Pre-service English as a foreign language teachers' perceptions of the relationship between multiple intelligences and foreign language learning. Learning And Individual Differences, 22(6), 850-855. https://doi.org/10.1016/j. lindif.2012.05.003

Schultz, D. P., \& Schultz, S. E. (2007). História da psicologia moderna. Thomson Learning.

Serin, N. B., Serin, O., Yavuz, M. A., \& Muhammedzade, B. (2009). The relationship between the primary teachers' teaching strategies and their strengths in Multiple Intelligences. Procedia Social and Behavioral Sciences, 1, 708-712. https:// doi.org/10.1016/j.sbspro.2009.01.124

Shearer, C. B. (2009). Criterion related validity of the MIDAS assessment. Image, 52(66), 1-33. https://www.bing.com/sear ch?q=Shearer $\% 2$ C + C. + B. $+\% 282009 \% 29 .+$ Criterion + related + validity + of + the + MIDAS + assessment. + Image $\% 2 \mathrm{C}+52 \% 28$ $66 \% 29 \% 2 \mathrm{C}+1-33 . \&$ form $=\mathrm{ANNH} 01 \&$ refig $=08 \mathrm{a} 96 \mathrm{a} 9 \mathrm{ee} 6 \mathrm{e} 74$ edecb3f377b61df3eef\&pc $=$ U531\&sp=-1\&pq $=$ shearer $\% 2 C+c$ .+b. $+\% 282009 \% 29 .+$ criterion + related + validity + of + the + mida $\mathrm{s}+$ assessment.+image $\% 2 \mathrm{C}+52 \% 2866 \% 29 \% 2 \mathrm{C}+1-33 . \& \mathrm{sc}=0$ $95 \& q \mathrm{~s}=\mathrm{n} \& \mathrm{sk}=\& \mathrm{cvid}=29 \mathrm{ac} 4 \mathrm{bda} 27 \mathrm{c} 54 \mathrm{~b} 57 \mathrm{~b} 2 \mathrm{~b} 1762 \mathrm{dba} 5 \mathrm{bd} 0 \mathrm{fa}$

Shearer, C. B. (2012). Cross cultural factor analytic studies of a Multiple Intelligences self-assessment. The International
Journal of Educational and Psychological Assessment, 12(1), 1-19. https://www.semanticscholar.org/paper/Cross-CulturalFactor-Analytic-Studies-of-a-Shearer/454c36cfa8b1d2e8278 $172 \mathrm{f} 477 \mathrm{~b} 2 \mathrm{~b} 7 \mathrm{~b} 2 \mathrm{e} 4 \mathrm{~d} 615 \mathrm{c} 6$

Sözen, H., Sözen, M., \& Tekat, A. (2009). Comparison of the profiles of the potential teachers in different disciplines based on multiple intelligences theory (Samsun City Sample). Procedia - Social and Behavioral Sciences, 1, 943-948. https:// doi.org/10.1016/j.sbspro.2009.01.167

Stipek, D., \& Gralinski, J. (1996). Children`s beliefs about intelligence and school performance. Journal of Educational Psychology, 88(3), 397-407. https://doi.org/10.1037/00220663.88.3.397

Teele, S. (1995). The Multiple Intelligences school (Ed. B. Bradley). Citrograph Printing.

Torresan, P. (2007). Intelligences and styles in language teaching: What is the difference? Didáctica (Lengua y Literatura), 9, https://core.ac.uk/download/38833553.pdf

Yalmanci, S. G., \& Gozum, A. I. (2013).The effects of multiple intelligence theory based teaching on students' achievement and retention of knowledge. International Journal on New Trends in Education and Their Implications, 4(3), 27-36. 04 http://www.ijonte.org/FileUpload/ks63207/File/04.yalmanci. pdf.

Yussen, S., \& Kane, P. (1985). Children's concept of intelligence. In S. Yussen, The growth of reflection in children (pp. 207-241). Academic Press.

Zandomeneghi, A. L. A. O. (2005). Ícones representativos das Inteligências Múltiplas: Uma proposta [Tese de doutorado, Universidade Federal de Santa Catarina]. Repositório Institucional UFSC. http://repositorio.ufsc.br/ handle/123456789/101866 